\title{
Content-Addressable Search Engines and DES-like Systems
}

\author{
Peter C. Wayner \\ Computer Science Department \\ Cornell University \\ Ithaca, NY 14853
}

\begin{abstract}
A very simple parailel architecture using a modified version of contentaddressable memory (CAM) can be used to cheaply and efficiently encipher and decipher data with DES-like systems. This paper will describe how to implement DES on these modified content-addressable memories at speeds approaching some of the better specialized hardware. This implementation is often much more attractive for system designers because the CAM can be reprogrammed to encrypt the data with other DES-like systems such as Khufu or perform system tasks like data compression or graphics.

The CAM memory architecture is also easily extendable to build a large scale engine for exhaustively searching the entire keyspace. This paper estimates that it will be possible to build a machine to test $2^{55}$ keys of DES in one day for $\$ 30$ million. This design is much less hypothetical than some of the others. in the literature because it is based upon hardware that will be available off-the-shelf in the late end of 1992. The architecture of this key search machine is much more attractive to an attacker because it is easily reprogrammable to handle modified DES-like algorithms such as the UNIX password system or Khufu.
\end{abstract}

The original DES system was designed to be easily implemented in hardware [NBSTT] and the current silicon manifestations of the cipher use modern processor design techniques to encipher and decipher information at about 1 to 30 megabits per second. Implementations of DES in software for standard CPUs, however, are markedly slower than specialized chips because many of the operations involved in DES are bit-level manipulations. As a result, many of the DES-like systems such as Merkle's Khufu [Mer90] were designed as replacements that could be easily implemented on conventional hardware.

There is one class of general architecture, however, that implements bit-level operations. The machines like the CM-1, CM-2 and CM-200 from Thinking Machines Corporation and the Maspar machine all have thousands of one-bit processors. The designers intended that a large number of processors would compensate for the deficencies of the individual nodes.

Another example of this small architecture is now emerging from the labs of memory designers who are trying to build sophisticated content addressable memory (CAM). The individual processors of these machines are even weaker than the ones of the CM-1, but they can be packed very densely on a chip. The tiny processors have only a fraction of the memory of a CM-1 (42 bits versus thousands) and only a one dimensional interconnection network (vs. 12), but this is sufficient to implement DES. Most importantly, these restrictions allow a packing density (1024 processors per chip) that is significantly higher at a cheap price. (\$30-\$100 per chip)

Implementing the cipher on generalized parallel architectures like the CAM have one main advantage- cost. Many computer designers often find that the speed of a specialized DES chip is often not worth the price. Generalized, content-addressable machines, however, have many other applications and this makes them a good compromise for the system designer. The design presented here can be easily reprogrammed in software to encrypt with DES or

E.F. Brickell (Ed.): Advances in Cryptology - CRYPTO '92, LNCS 740, pp. 575-586, 1993.

(C) Springer-Verlag Berlin Heidelberg 1993 
any DES-like variant like Khufu. The hardware can also be used do data compression, data searches or even many different graphics operations.

This paper will describe how to implement the DES algorithm on this architecture and produce results that are on par with the middle range of the specialized hardware. The main contribution is not extremely fast encryption speeds. It is very fast speed coupled with software-level flexibility. Many other papers have offered flexib]e hardware designs [VHVM88, FMP85] that can be easily reworked to handle variants of DES, but none offer the flexibility of this system. Verbauwhede et al. [VHVM88] requires new silicon to be fabricated in all cases and the designs of Falfield et al. [FMP85] run internal microcode that can be easily reprogrammed to implement other slight variants of DES such as cipher-block chaining. However, new algorithms like Khufu, however, would require a new micro-code instruction set. The flexibility of this CAM based design is quite attractive to both the system designer and the brute-force attacker because it allows the hardware to be used for different purposes and different algorithms.

\section{Content-Addressable Memory Machines}

Standard memory maps an address to a value. Unfortunately, there are many applications when an algorithm needs to know which memory location holds a particular value. The only recourse is to search all the memory to find the value in question. Content-addressable memory is a hardware solution to this problem that will invert the search and provide the address bolding a value in a single operation. This technique has been well-researched over the years and the book by Kohonen [Koh87] notes many approaches and summarizes some of the more salient aspects of this research. Several companies including AMD are making basic content-addressable memory modules.

Recently teams at Syracuse University (some publications include (Old86, OW 187 , OSB87]), MIT and Cornell ([Bri90, WS89. Zip90]) have developed more sophicated and powerful implementations in silicon. These implementations allow the programmer to chain the result of several searches together in a simple fashion so that larger data structures and more complicated searches can be performed in hardware. Some of this hardware was originally intended to speed up logic programming, but many people have found surprising and interesting applications for the simple hardware. Oldfield and his team at Syracuse, for instance, are currently working on compressing data.

A company, Coherent Research Incorporated of Syracuse, Sew York, is building sophisticated content-addressable memory chips called the Coherent Processor for widespread use. This paper will use their chip as an example because it is commercially available, but there is no reason why the algorithms cannot be modified slightly for use on similar chips.

At the basic level, the Coherent Processor is a large, single dimensional array of very simple parallel processors. Each processor has 42 bits of memory $\left(W_{i}[0] \ldots W_{i}[41]\right.$, the $i$ denotes the processor number) and three one-bit registers $\left(R_{1}, R_{2}\right.$ and $\left.R_{3}\right)$. It also has a processing unit that can execute instructions on the registers, transfer data between the registers and the memory, communicate with the two neighboring processors or match a value on the internal bus. The instructions are simple operations that read the three register bits of memory and store the result in one of the three. The match instructions can be used to simultaneously compare one 42 -bit value against the entire array of processors. If there is a match, then the appropriate value is placed in a register.

The following table shows the basic Coherent Processor instructions and the number of clock cycles used to complete them.

1. MATCH: Simultaneously compare the 42 general bits at each processor with the values on a bus and store the result of this match in $R_{1}$. This is used to look up items quickly. 
The match routine can include wild-card matches for individual bits so it is possible to match for strings of bits like " $0000^{* * * * * *} 11^{* * * * * " ~(~} a$ "*" matches both a " 0 " and a " 1 "). If you want to move the value of bit $W_{i}[2]$ into $R_{3}$, then you would "match" a pattern with 1 in bit $W_{i}[2]$ and wild-card matches specified for the rest and store the result in $R_{3}$. If the value of bit $W_{i}[2]$ was 1 in a particular word, then the match would be successful and a $I$ would be stored in $R_{3}$. If a zero was in bit $W_{i}[2]$, then the match would be unsuccessful and a zero would be stored. The values of the other columns would not be affected. Cost: 4 cycles.

2. CALC: Calculate a three-bit function of the three registers and store the result in a third register. Cost: 2 cycles.

3. READ: Take the result of a selected word and place it on the bus. This operation usually follows a MATCH operation. Cost: 3 cycles.

4. WRITE: Move the result from the bus into the selected word(s). Cost: 2 cycles.

5. SHIFT: The first registers of each word are interconnected. They can shift the bit in their register to adjacent words in one step. Cost: 2 cycles.

6. WRITECOLUMN: Hoves a bit from a register into one of the 42 bits of memory. Cost: 2 cycles.

These commands can be strung together to manipulate data in simple and straightforward methods.

\section{Implementing Plain DES}

There are three main operations involved in encrypting a block of 64 bits with the basic mode of the Data Encryption Standard known as the Electronic Code Book (ECB). They are 1) permuting the bits, 2) passing a 32-bit block through an s-box and 3) permuting the key structure. Each of these steps is easy to program on the Coherent Processor, in a large part because the architecture is so limited. Several features of the instruction set, however, make implementing the algorithm very easy.

Let the plaintext blocks of data be denoted, $B_{2}, \ldots, B_{n}$ and the individual bits of block $B_{i}$ be $\left\{B_{i}[0] \ldots B_{i}[63]\right\}$. The key is $K$ and the individual bits are $K[0] \ldots K[55]$.

There are sixteen rounds of encryption and the key scheduling algorithm chooses a 48 -bit subset of key bits to be used on each round. Let $K^{(7)}[0] \ldots K^{(i)}[47]$ be the 48 bits used in round $l$. Each block of 64 bits is broken into two 32 -bit halves (called $B_{L}$ and $B_{R}$ ) and in each round the value of one of the halves is mixed with a subset of the key bits, passed through the s-box and then mixed with the right 32-bit balf. More precisely, in each round:

$$
B_{L}-B_{L} \oplus f\left(E\left(B_{R}\right) \oplus K^{(n)}\right) .
$$

(" $\oplus$ " =XOR) Then $B_{L}$ and $B_{R}$ are exchanged. $f$ is the s-box function that takes 48 bits and returns 32 and the $E()$ function is an "expansion" function that maps 32 bits into 48 bits so it can be combined with the 48 bits of key. Some bits of the input to $E$ are used more than others.

The data to be encrypted is broken into 64-bit blocks and each block is stored in 32-bit halves in two adjacent 42 bit words in the array, $W_{i}$ and $W_{i+1}$.

\subsection{Permuting the Bits}

At the beginning and the end of the encryption process, the 64 bits in the block are passed through a bit-wise permutation. This step is often considered the slowest part of many software implementations for general purpose machines and many people believe that it was 
included to slow down software implementations and force general CPUs to move bits one by one. The Coherent Processor must also move each bit one at a time, but at least this is the best that it can do. In practice, the large number of parallel processors makes up for the weakness.

Let the permutation be written as a set of cycles: $W_{i}\left[p_{0}\right] \rightarrow W_{j}\left[p_{1}\right] \rightarrow \ldots \rightarrow W_{i}\left[p_{i}\right] \rightarrow$ $W_{i}\left[p_{0}\right]$. There are 64 bits to be exchanged, but they do not move in one cycle. The process can be accomplished by stringing together a chain of bit moving commands. When the bits to be exchanged are on different words, then the CAM must also execute a bit-passing command to swap the bit to the adjacent word. The work can be summarized in pseudo-code:

Move $W_{i}\left[p_{0}\right]$ into a bit .

for $\mathrm{k}:=1$ to 63 do

Move $W_{i}\left[p_{k}\right]$ into a bit.

Move $W_{i}\left[p_{k-1}\right]$ into its destination.

If $W_{i}\left[p_{k}\right]$ is on the wrong word,

then pass it to the correct one.

Move $W_{i}\left[p_{63}\right]$ into $W_{i}\left[p_{0}\right]$.

There are only 32 bits that need to be shifted between words. It is possible to do this quickly. The next section which computes the values of the s-boxes is much more time intensive. The cost: $129 \mathrm{MATCH}$ and WRITECOLLMN instructions, 32 SHIFT instructions. About 580 cycles.

\subsection{Computing the S-boxes}

The s-box are responsible for providing the non-linear mixing of the bits that is necessary to provide adequate security. At the highest level. the $\mathrm{s}$-box is a function that maps 32 bits to 32 other bits. The s-boxes used in DES are, though, much simpler and they can be described as eight functions that take 6 out of the 32 bits and return four. Some bits are used more than others. These eight s-boxes can be further simplified into 32 functions that map six bits to one bit and this is the best level of abstraction to use when programming the Coherent Processor.

Meyer and Matyas [MM82] describe the design of the s-boxes in terms of minterms, which are roughly the same as clauses of boolean variables. An equation describing output of one bit of an s-box might look something like this:

$$
B_{i}[1] \cdot \neg B_{i}[2] \cdot B_{i}[3] \cdot B_{i}[4]+B_{i}[1] \cdot \neg B_{i}[5] \cdot-B_{i}[6]+B_{i}[2] \cdot B_{i}[5] .
$$

(" "=boolean and, " $f$ "=boolean or, " $\neg "=$ boolean not.) There are three minterms in the example and it is generally believed that the number of minterms in a minimal expression is one measure the complexity of the s-box. The recert papers by Biham and Shamir [BS91] and others, show that there are additional criterion that are more important. Meyer and Matyas note that there are 52 and 53 minterms in the description of each of the 8 s-boxes.

These minterm descriptions of the s-boxes can be directly converted into operations for the Coherent Processor. Each clause of variables to be ANDed together can be computed with a MATCH equation with appropriate set of ones for the variables in the clause, zeros for the negated variables in the clause and wildcards for the unrepresented variables. The expression from equation 1 can be encoded: 


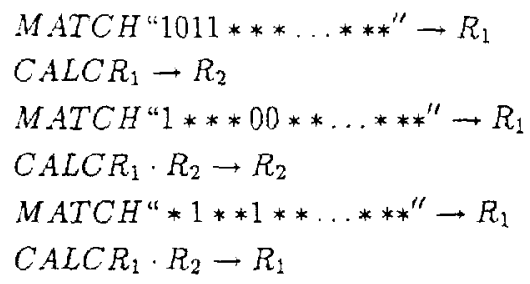

This takes 6 cycles per minterm. At 53 minterms per s-box and 8 s-boxes per encryption round, this takes 2544 cycles per encryption round to calculate the values of the bits. It takes one SHIFT, one MATCH, one CALC and one COLUMNWRITE to XOR each of the 32 bits into the adjacent word. That is an additional 384 cycles for 2928 per encryption round. There are 16 rounds in DES, the permutations take 580 cycles and the overall encryption process takes 47,528 cycles.

\subsection{Handling the Key}

When the result of one of the 32 functions is computed it must be XOR-ed with the key and then passed to the adjacent word to be XOR-ed with the appropriate bit. The same key encrypts all the blocks at the same time and it can be included by XORing the key vector, $K^{(I)}$, into the match words. For instance, assume that "110011001010111001001100 11100101 " is the 48 bits of key being used in a round and the minterms from equation 1 define the s-box equations. Then the operations in example 2 become:

$$
\begin{aligned}
& M A T C H " 0111 * * * \ldots * * * " \rightarrow R_{1} \\
& C A L C R_{1} \rightarrow R_{2} \\
& M \operatorname{ATCH} " 0 * * * 11 * * \ldots * * * "-R_{1} \\
& C A L C R_{1} \cdot R_{2} \rightarrow R_{2} \\
& M A T C H * 0 * * 0 * * \ldots * * * "-R_{1} \\
& C A L C R_{1} \cdot R_{2} \rightarrow R_{1}
\end{aligned}
$$

The same key is used to encrypt or decrypt each block of data in the simple version of DES. There are $56 \mathrm{key}$ bits, but only 48 of them are used during each of the 16 different rounds. The bits being used are maintained by the program running on the general machine that is driving the Coherent Processor. It selects the subset of 48 bits that are used in each encryption and modifies the s-box functions accordingly.

This method presupposes that the sixteen 48 -bit subsets of the keys are precomputed and "compiled" into the code. This process is non-trivial and certain to cost some time. When the amount of data encrypted or decrypted per key change is large, then this "compilation" time is minimal. If the key is changed frequently, then there may be some impact on the encryption times. It is not likely to impact the overall throughput, however, if the CPU driving the CAM array is fast enough to interleave operations in between the various CAM instructions. This is not unreasonable because many of the CAM instructions take 2 to 4 cycles to complete. A modern pipelined RISC architecture should be able to complete the key scheduling instruction inbetween. A better understanding of the effects of this will need to wait until the software is completely implemented on a working system. 


\subsection{The Total Cost}

The current version of the Coherent Processor will run at speeds up to $50 \mathrm{MHtz}$. If an encryption takes about 47,428 cycles, then each pair of words in the processor array can encrypt about 1,000 64-bit blocks per second. Writing a word into the array and reading it out takes 5 cycles in total. One chip of the current model has 1024 words or processors, so it can read in, encrypt and write out blocks of $32 \mathrm{~K}$ in 52,548 cycles. This is equivalent to 31.2 megabits per second-something that is in line with the middle range of current DES chips. The Cryptech CRY12C102 data sheet reports that it runs at 22.5 megabits per second and the Pijnenburg PCC100 attains 20 megabits per second. Moreover, the Coherent Processor is designed to be easily expanded by linking together multiple copies of the chip and $n$ chips will $n$ times faster for small numbers of $n$. When there are hundreads or thousands of chips, the cost of writing and reading the information from the Coherent Processor becomes the limiting factor. Coherent Research reports that the new chip will cost about $\$ 100$ per copy in small quantities and substantially less in large ones.

\section{Exhaustive Attack on DES}

When DES was introduced in 1977: some computer scientists protested that 56 bits were not sufficient because it would be possible to do an exhaustive search of the key space in a short amount of time using a massively parallel computer. In their book, Meyer and Matyas [MM82] discount that possiblity and predict that it would just not be physically possible to build the machine until the 1990's because there were too many physical linitations. Heat and power usage are two major barriers. Diffe and Hellman describe the design in detail and respond to these criticism in [DH77].

How easy would it be to build one today? Standard off-the-shelf encryption chips are plentiful and relatively cheap, but they require a second processor feeding them the keys and the test cases. Anyone who wants to build such a machine must undertake a project of building such a large array of distributed computers. This would require a large amount of custom design work. A truly dedicated attacker could even fabricate custom DES testing chips which have a built in circuit for incrementing the key by one bit and testing the result against another register. Only governments could afford a budget this large. Moreover, the slightest change in the algorithm would render this machine worthless.

Garon and Outerbridge calculated the approximate costs of designing such a machine and found that it would cost about $\$ 129,000$ for a machine that would break DES within 1 year if the machine was built in 1990. [GO91]. They also say that a machine that could exhaustively search all the bits in one day for $\$ 46$ million in 1990 . This price would drop to $\$ 18$ million in 1995. They assume that it is possible to build a node that encrypts 2 million key tests for $\$ 25$ in 1990 in order to complete such a machine. They do not describe the details of how to design the board or manufacture it is sufficient quanties.

The Content Addressable Memory array chips, however, are designed to be built into large parallel arrays of chips. It is already possible to buy a board for a PC which has 64 chips of a previous model of the Coherent Processor. Large arrays should not be hard to create. Moreover, the algorithm is implemented in software, so the machine can also be used to attack many other subtle and not-so-subtle variations of DES.

What is the best way to do an exhaustive search with the current architecture of the Coherent Processor? The version described for simple encryption and decryption is able to work very quickly because it can encode the key in the stream of instructions fed to the Coherent Processor. This approach must be abandoned because an exhaustive search of the key space requires that each processing node must use a different key. 
One alternative is to store the key bits in the 10 extra tag bits stored at each node. Two nodes are used to hold the two 32-bit half-blocks of each case, so there are up to 20 extra key bits which can be stored at each node. Let there be $2^{n}$ processors in the machine. That means there are $2^{n-1}$ potential keys that can be tested with each round because two nodes are used for each encryption. Assume that $n \leq 21$ and the problem does not overflow the physical space of the real machine. (Later versions of the architecture could have more free bits available.) At each pair of nodes, store a unique set of $n-1$ key bits. These bits will be used by this pair of nodes alone. The other $56-(n-1)$ bits are shared by all the instances and they are encoded in the instruction stream as before.

At the beginning of each round of encryption, the local key bits must be XOR-ed into the appropriate balf-block of bits before that half-block is passed through the s-boxes. These four or five instructions will XOR in the key bit $K_{\mathrm{i}}$ in to position $B_{j}$ :

$$
\begin{aligned}
& \text { MATCHKi } \rightarrow R_{1} \\
& \text { SHIFT } \\
& \text { MATCHB; } \rightarrow R_{2} \\
& \text { CALCR XORR } \rightarrow R_{2} \\
& \text { WRITECOLUMYR } \rightarrow R_{3}
\end{aligned}
$$

The SHIFT instruction is only necessary if the key bit is on the opposite node from the destination bit. This process is repeated at the end of the s-box calculation to remove the bit from the data. Only 48 of the 36 key bits are used at each round, but it is possible that up to $n-1$ of these bits will come from the bits stored locally. The operations in equation 4 take 16 cycles. They must be repeated $2 n-2$ times for each round. The result takes $512 n-512$ extra cycles for each encryption. If a machine was built with a full complement of $2^{21}$ processors, then it would take 57,126 cycles to test $2^{20}$ potential heys. This step must be repeated $2^{36}$ times and the machine is capable of doing about 875 of these tests per second or about 76 million per day. Exhausting the entire space would require 904 days. If the well-known trick of exploiting symmetry in the keys is used to reduce the key space to $2^{55}$ keys, then one machine will test all in 452 days.

How much would such a machine cost? There are $2^{10}$ processors on a chip that will cost between $\$ 30$ and $\$ 100.2^{11}$ chips are necessary and this would cost between about $\$ 60,000$ and $\$ 200,000$. Control hardware would add additional $\$ 10,000$ to $\$ 20,000$. 45 machines would cost about $\$ 3$ million dollars and exhaustively search the space in 10 days. $\$ 30$ million would buy a machine that would search the space in 1 day with 450 machines. I'm assuming that volume discounts would apply at this scale and $\$ 30$ is a price that should apply at the end of 1992 when the chips become widely available.

Although this design is still hypothetical, it is much more real than some of the other designs available because the chip fabrication and design is already complete. The process of building a machine out of chips is not much different from connecsing a large bank of memory up to a single processor. This paper does not pretend to addresss any of the important questions about heat and power dissipation. These could also affect the design and it is possible that my estimate of $\$ 10,000$ to $\$ 20,000$ for the support hardware is too low.

The standard assumptions about time and transistor density should apply to this model as well. It is entirely conceivable that we will see larger improvements in density and price of these machines in the near future because they are younger designs.

The UNIX password system uses a version of DES that was presumably modified to make it impossible to gang together a number of off-the-shelf DES chips and use the system to 
break UNIX passwords. This large machine, however, is not constrained by this modification or any other modification that re-arranges the pattern of s-boxes, permutations and mixing. The salting process used in the UNIX password operation is easy to express with extra bit swapping operations. The only problem with attacking systems like Merkle's Khufu is expressing the s-boxes as minterms. Incidentally, logic minimization is also easily handled by the Coherent Processor.

The availability of these systems puts even more pressure on the Unix password system. In 1989, Feldmeier and Karn [FK89] estimated that the UNIX password system was insecure for short alphanumeric passwords because a DEC 3100 could process about 1000 passwords per seconds. Given that each password needs 25 passes of DES, then it is possible to estimate that a Coherent Processor based processor will be able to test about 20,000 passwords per second per chip. If a basic Coherent Processor processor comes with between 8 to 64 chips, then it is easily possible to imagine computers with the ability to test between 160,000 and $1,280,000$ tests per second. How fast could such a standard machine test all passwords made up of 6 alphanumeric characters ("A" - "Z", "a" - " $z$ ", " 1 " - " 9 ") ? Between about 3.75 days ( 8 chips) and about half a day (64 chips). A large scale machine with $2^{1} 1$ chips should be able to tackle passwords with 7 alphanumeric passwords in about one day.

\section{DES with Modified Chaining}

The last several sections described how to encrypt a large block of data in parallel using a simple DES with no feedback. A more robust version of DES feeds the result of encrypting each block into the key selection of the next block. Let $E_{i}=f\left(K, B_{i}\right)$ represents the ciphertext blocks. $A$ feedback cipher sets $E_{i}=f\left(K, B_{i} \oplus E_{i-1}\right)$ where " $\oplus$ " represents boolean XOR. $E_{0}$ is set to a pre-arranged constant. This process is called Cipher Block Chaining (CBC).

The modification adds a great deal of strength to the plain DES because it reduces the redundancies that can developed if there is an 64 bit block that occurs often in the plaintext. The feedback mode ensures that a different value will permute each block and obscure the redundancy. It should be obvious that this system cannot be used when all the blocks are computed in parallel. Here is a modified version of chaining that can be implemented in parallel.

One solution is to exchange and XOR bits with neighbors at the end of certain rounds of encryption. In round 1, the left half of each block is used to compute the value XORed into the right half. After this, the left blocks are exchanged with the neighboring blocks and XOR'ed into the right halves of the neighboring block. This can be done with pseudo-code like this. $W_{i}$ is the left half and $W_{i+1}$ is the right half.

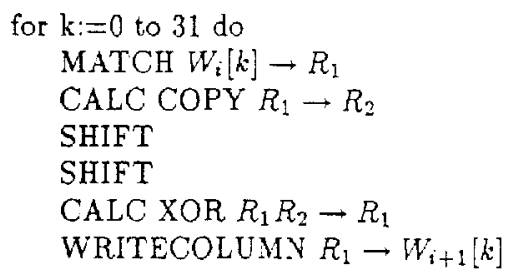

This command shifts one bit to the next pair of words over and XOR's it with the value of a neighboring block. It takes 16 cycles per bit to achieve this. This can be repeated as often as desired at the cost of slowing down the entire encryption. Doing this at the end of each round of encryption costs 8,192 cycles and this slows the encryption rate to 27.0 megabits 
per second. In this case, a change in block $B_{i}$ will propigate through blocks $B_{i}$ to $B_{i+16}$ and effect their encrypted values. Arbitrarily complex shifting can be included as long as care is taken to ensure that the results can be reversed. If this step is done often in the process, it can effectively turns the encryption into one large block at a small decrease in speed.

\section{Conclusion}

This paper has described a simple architecture intended for information storage and retrieval that can also encrypt and decrypt messages faster than all but the best specialized chips. More importantly, the results are achieved in software so the process can be extended to other DES-like systems without refabricating the chips. The only problem is expressing the s-boxes so they can be implemented with minterms. This should make the chip much more desirable for many implementations of DES that require more flexibility than extreme speed.

Chips like the Coherent Processor also make it very easy to create a large-scale processor for exhaustive cryptanalysis of the key space because the chips were designed to be grouped together in a large array. The hypothetical machine described here is much different from the other machines described in the literature because it is both reprogrammable and substantially closer to being realized. Only a minimal amount of logic is necessary to turn the chips into machines that are able to handle DES and variants of DES like the UNIX password system or Khufu.

The flexible software structure also provides an easy method to test for broken chips. It is possible to load each line with a test vector, encrypt them in parallel and then test for failures with a MATCH instruction. Many of the earlier designs for large machines needed to build in a specific test function to maintain the system.

There are several changes to the Coherent Processor that would improve its ability to encrypt DES. Currently, the key is "compiled" into the program for the CAM and this may be a non-trivial event. If future versions of the architecture have more that 42 bits per word, then it could be practical to store the key locally and add the key in bit by bit as it is done in the brute force attack. Also, the current version of the Coherent Processor will only compute 3 bit functions. 4 or 5 bit functions may be quite practical and they would certainly speed the results of the process.

Working hardware is due in early 1993 and this will provide an opportunity to develop

\section{Acknowledgements}

The author would like to thank Chuck Stormon at Coherent Research for taking the time to teach me how to program the Coherent Processor and making many valuable comments about the structure of this paper. I would also like to thank Luke $O^{\prime}$ Connor for his comments on the structure of the paper and for providing the minterm representations of the s-box included here in the appendix. Richard Outerbridge also provided invaluable help and suggestions.

\section{References}

[Bri90] Sharon Marie Britton. 8k-trit Dotabase Accelerator with Error Detection. PhD thesis, Massachusetts Institute of Technology, February 1990.

[BS91] Eli Biham and Adi Shamir. Differential cryptanalysis of Snefru, Khafre, REDOC-Il, LOKI, and lucifer. In Crypto 91, Santa Barbara, California, 1991.

[DH77] Whitfield Diffie and Martin Hellman. Exhaustive cryptanalysis of the nbs data encryption standard. Computer, 10(6):74-84, 1977. 
[FK89] David C. Feldmejer and Philip R. Karn. Lnix password security- ten years later. In G. Brassard, editor, Advances in Cryptology: Proceedings of Crypto '89, pages 44-63, New York City, Berlin, 1989. Springer-Verlag.

[FMP85] Robert C. Fairfield, Alex Matusevich, and Joseph Plany. An lsi digital encryption processor. IEEE Communication, pages 23-27, July 1985.

[GO91] Gilles Garon and Richard Outerbridge. Des watch: And examination of the sufficiency of the data encryption standard for financial institution's information security in the 1990's. Cryptologia, 15(3):177-193, July 1991.

[Koh87] Teuvo Kohonen. Content-Addressable Memories. Springer-Verlag, Berlin, New York City, 1987.

[Mer90] Ralph Merkle. Fast software encryption function. In A.J. Menezes and S.A. Van Stone, editors, Crypto 90, Berlin, New York City, 1990. Springer Verlag.

[MM82] Carl H. Meyer and Stephen M. Matyas. Cryptography: New Dimension in Computer Security. John Wiley and Sons, New York, 1982.

[NBSi7] NBS. Data encryption standard (des). Technical report, National Bureau of Standards (US), Federal Information Processing Standards, Publication 46, National Technical Information Services, Springfield, Virginia, April 1977.

[Old86] J.V. Oldfield. Logic programs and an experimental architecture for their execution. Pracedings of the I.E.E.E. Part E, 133:163-167, 1986.

[OSB87] J.V. Oldfield, Charles D. Stormon, and M.R. Brule. The application of vlsi contentaddressable memories to the acceleration of logic programming systems. In CompEuro 87, VLSI and Computers, pages 27-30, Hamburg, Germany, May $198 i$.

[OWN87] J.V. Oldfield, R.D. Williams, and N.E.Wiseman. Content-addressable memories for storing and processing recursively-divided images and trees. Electronics Letters, 23(6):262$263,1987$.

[ST79] Robert Morris Sr, and Ken Thompson. Password security: A case history. Communications of the ACM, 22:594-597, Navember 1979.

[VHVM88] Ingrid Verbauwhede, Frank Hoornaert, Joos Vandewalle, and Hugo J. De Man. Security and performance optimization of a rew des encryption chip. IEEE Journal of Solid-State Circuits, pages 647-656, June 1988.

[WS89] John Wade and Charles Sodini. A ternary content-addressable search engine. IEEE Journal of Solid-State Circuits, 24(4):1003-1013, August 1989.

[Zip90] Richard Zippel. Programming the data structure accelerator. In Proceedings of Jerusalem Conference on Information, Technology, Jerusalem, Israel, October 1990.

\section{Appendix}

Some minimal representations of the s-boxes provided by Luke O'Connor. $S_{1}^{2}$ represents the function for the first bit of the second s-box. The " + " means means logical or. The logical ands in each clause (implicant) are left out to save space. A variable with a bar over it " $\overline{x_{6}}$ ") represents NOT $x_{6}$.

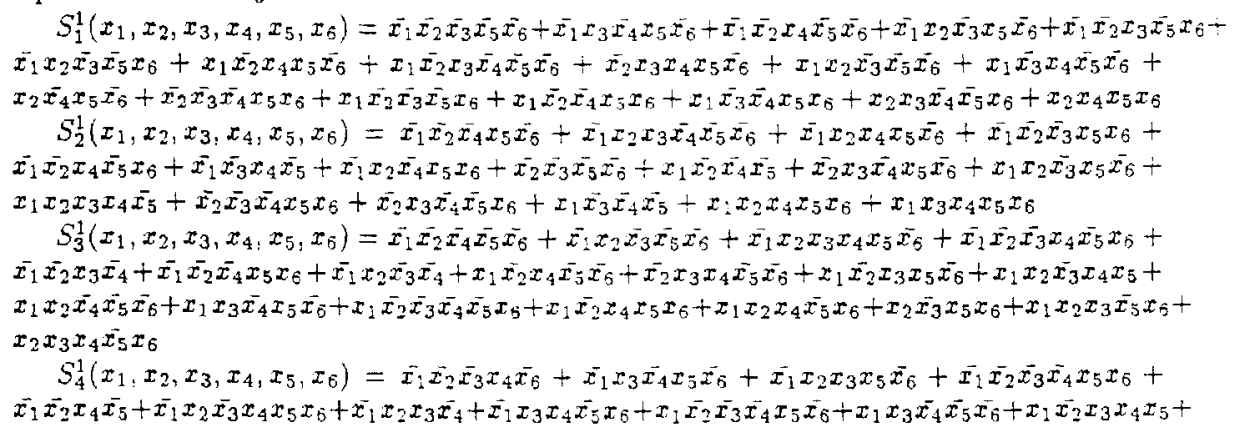


$x_{1} x_{2} \overrightarrow{x_{3}} x_{4} \overline{x_{6}}+x_{2} \overline{x_{4}} \overrightarrow{x_{5}} \overline{x_{6}}+x_{1} x_{2} \overline{x_{5}} \bar{x}_{6}+x_{1} \overrightarrow{x_{2}} x_{3} x_{5} x_{6}+\overrightarrow{x_{2}} x_{3} x_{4} x_{6}+x_{1} \overline{x_{3}} \overline{x_{4}} \overline{x_{5}} x_{6}+x_{1} x_{2} \overline{x_{3}} \overline{x_{4}} x_{6}+$ $x_{1} x_{2} \overline{x_{3}} \overline{x_{5}}+x_{1} x_{3} x_{4} x_{5} x_{5}$

$S_{1}^{2}\left(x_{1}, x_{2}, x_{3}, x_{4}, x_{5}, x_{6}\right)=\overline{x_{1}} \overline{x_{2}} \overline{x_{3}} \overline{x_{5}} \overline{x_{6}}+\overline{x_{1}} \overline{x_{2}} x_{3} \overline{x_{4} x_{5}} \overline{x_{6}}+\overline{x_{1}} \overline{x_{3}} \overline{x_{4}} \overline{x_{5}} \overline{x_{6}}+\overline{x_{1}} \overline{x_{3}} x_{4} x_{5} \overline{x_{6}}+$ $\overrightarrow{x_{1}} \bar{x}_{2} x_{3} \overrightarrow{x_{5}} x_{6}+\overline{x_{1}} \overline{x_{2}} x_{3} x_{4} x_{6}+\overline{x_{1}} x_{2} x_{3} \overline{x_{4}} x_{5} x_{6}+\overline{x_{2}} \overline{x_{3}} x_{4} x_{5} \overrightarrow{x_{6}}+\overline{x_{1}} \overline{x_{2}} x_{3} \overline{x_{5}} \overline{x_{6}}+\overline{x_{1}} \overline{x_{3}} \overline{x_{4}} x_{5} \bar{x}_{6}+$ $x_{1} x_{2} \overline{x_{3} x_{4}} \overline{x_{5}} \overline{x_{6}}+x_{2} x_{3} \overline{x_{4}} \overline{x_{5}} \overline{x_{6}}+x_{2} x_{3} x_{4} x_{5} \overline{x_{6}}+\overline{x_{2}} \overline{x_{3}} \overline{x_{4}} x_{5} x_{6}+x_{1} \overline{x_{2}} \overline{x_{3}} \overline{x_{5}} x_{6}+\overline{x_{1}} \overline{x_{2}} \overline{x_{4}} x_{5} x_{6}-$

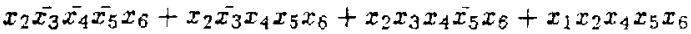

$S_{2}^{2}\left(x_{1}, x_{2}, x_{3}, x_{4}, x_{5}, x_{6}\right)=\overline{x_{1}} \overline{x_{2}} \overline{x_{4}} \overline{x_{5}} \overline{x_{6}}+\overline{x_{1}} x_{2} \overline{x_{3}} x_{5} \overrightarrow{x_{6}}+\overline{x_{1}} x_{2} x_{3} \overline{x_{5}} \overline{x_{6}}+\overline{x_{1}} \overline{x_{2}} \overline{x_{3}} x_{5} x_{6}+\overline{x_{1}} \overrightarrow{x_{2}} \overline{x_{3}} x_{4} x_{6}+$ $\overline{x_{1}} \overline{x_{2}} x_{4} x_{5}+\overline{x_{1}} x_{2} \overline{x_{4}} \overline{x_{5}} x_{6}+\overline{x_{1}} x_{3} \overline{x_{4}} \overline{x_{5}}+\overline{x_{1}} x_{3} x_{4} x_{5} x_{6}+x_{1} \overline{x_{2}} \overline{x_{4}} x_{5} \overline{x_{6}}+x_{1} \overline{x_{2}} \bar{x}_{4} \overline{x_{5}} \overline{x_{6}}+x_{1} x_{2} \overline{x_{3}} \overline{x_{5}} \overline{x_{6}}+$ $x_{1} x_{2} x_{4} x_{5} \overline{x_{6}}+x_{1} \overline{x_{2}} \overline{x_{3}} \overline{x_{4}} \overline{x_{5}} x_{6}+x_{1} x_{2} x_{4} \overline{x_{5}} x_{6}+x_{1} x_{2} \overline{x_{3}} x_{5} x_{6}+x_{1} x_{3} \overline{x_{4}} x_{5} x_{6}+x_{1} x_{3} x_{4} \overrightarrow{x_{5}} x_{6}$

$S_{3}^{2}\left(x_{1}, x_{2}, x_{3}, x_{4}, x_{5}, x_{6}\right)=\overline{x_{1}} \overline{x_{2}} x_{3} \overline{x_{4}}+\overline{x_{1}} \overline{x_{2}} x_{3} \overline{x_{5}} \overline{x_{6}}+\overline{x_{1}} x_{2} \overline{x_{3}} \overline{x_{4}} x_{5} \overline{x_{6}}+\overline{x_{1}} \overline{x_{2}} \overline{x_{3}} x_{4} \overline{x_{5}} \overline{x_{6}}+$ $\overline{x_{1}} \overline{x_{2}} \overline{x_{4}} \overline{x_{5}}+\overline{x_{1}} \overline{x_{3}} x_{4} x_{5} x_{6}+\overline{x_{1}} x_{3} \overline{x_{4}} \overline{x_{5}} x_{6}+x_{1} \overline{x_{2}} \overline{x_{3}} x_{5} \overline{x_{6}}+x_{1} \overline{x_{2}} \overline{x_{3}} x_{4} \overline{x_{6}}+\overline{x_{2}} \overline{x_{3}} x_{4} x_{5} \overline{x_{6}}+\overline{x_{2}} \overline{x_{3}} \overline{x_{4}} \overline{x_{5}}+$ $x_{1} \overline{x_{3}} x_{4} x_{5} \overline{x_{6}}+x_{1} x_{2} x_{3} x_{5} \overline{x_{6}}+x_{1} x_{2} x_{3} x_{4} \overline{x_{6}}+x_{2} x_{3} x_{4} x_{5} \overline{x_{5}}+\bar{x}_{2} x_{3} x_{5} x_{6}+x_{1} x_{2} \overline{x_{3}} \overline{x_{4}} x_{6}+x_{1} \overline{x_{3}} x_{4} \overline{x_{5}} x_{6}+$ $x_{2} x_{3} x_{4} \bar{x}_{5} x_{6}$

$S_{4}^{2}\left(x_{1}, x_{2}, x_{3}, x_{4}, x_{5}, x_{6}\right)=\overrightarrow{x_{1}} \overline{x_{2}} \overrightarrow{x_{4}} x_{5} \overrightarrow{x_{6}}+\overline{x_{1}} \overline{x_{3}} \overline{x_{4}} \overline{x_{6}}+\overrightarrow{x_{1}} x_{2} \overline{x_{3}} x_{5} \overline{x_{6}}+\overline{x_{1}} x_{3} x_{4} \overline{x_{5}} \overline{x_{6}}+\overline{x_{1}} \overline{x_{2}} \overline{x_{3}} x_{5} x_{6}+$ $\overline{x_{1}} x_{2} x_{4} \overline{x_{5}} x_{6}+x_{1} \overline{x_{2}} x_{4} \overline{x_{6}}+x_{1} x_{2} \overline{x_{1}} \overline{x_{5}} \overline{x_{6}}+x_{1} x_{2} x_{3} x_{5}+\overline{x_{2}} \overline{x_{3}} x_{4} x_{5} x_{6}+\overline{x_{2}} \overline{x_{4}} \overline{x_{5}} x_{6}+x_{1} x_{3} \overline{x_{4}} x_{5} x_{6}+$ $x_{1} \vec{x}_{3} \bar{x}_{4} \overline{x_{5}} x_{6}+x_{2} \overline{x_{3}} x_{4} \overline{x_{5}} x_{6}+x_{2} x_{3} x_{5} x_{6}$

$S_{1}^{3}\left(x_{1}, x_{2}, x_{3}, x_{4}, x_{5}, x_{6}\right)=\overline{x_{1}} \overline{x_{2}} x_{4} \overline{x_{5}} \overline{x_{6}}+\overline{x_{1}} x_{2} \overline{x_{3}} \overline{x_{4}} x_{5}+\overline{x_{1}} \overline{x_{3}} x_{4} \overline{x_{5}} \overline{x_{6}}+\overline{x_{1}} x_{2} x_{3} \overline{x_{4}} \overline{x_{5}}+\overline{x_{1}} x_{2} x_{3} x_{4} x_{5} \overline{x_{6}}+$ $\overline{x_{1}} \overline{x_{2}} \overline{x_{3}} \overrightarrow{x_{4}} \overline{x_{5}}+\overline{x_{1}} \overline{x_{2}} x_{4} x_{5} \bar{x}_{6}+\overline{x_{1}} \overline{x_{3}} x_{4} x_{5} x_{6}+\overline{x_{1}} x_{2} \overline{x_{4}} x_{5} x_{6}+\overline{x_{1}} x_{2} x_{3} \bar{x}_{6} x_{6}+\bar{x}_{2} \overline{x_{3}} \bar{x}_{4} x_{5} \overline{x_{6}}+x_{1} \overline{x_{2}} \overline{x_{4}} \overline{x_{5}} \overline{x_{6}}+$ $x_{1} \overline{x_{3}} \overline{x_{4}} \overline{x_{5}} \overline{x_{6}}+x_{1} \overline{x_{3}} x_{4} x_{5} \overline{x_{6}}+x_{1} x_{3} \overline{x_{4}} x_{5} \overline{x_{5}}+x_{1} x_{2} x_{3} x_{4} \overline{x_{5}} \overline{x_{6}}+x_{1} \overline{x_{2}} \overline{x_{4}} x_{5} x_{6}+x_{1} \overline{x_{2}} x_{4} \overline{x_{5}} x_{6}+$ $x_{2} \vec{x}_{3} \bar{x}_{4} x_{5} x_{6}+x_{1} \bar{x}_{3} x_{4} \vec{x}_{5} x_{6}+x_{2} x_{3} \bar{x}_{4} \bar{x}_{5} x_{6}+x_{1} x_{2} x_{3} x_{4} x_{5} x_{6}$

$S_{2}^{3}\left(x_{1}, x_{2}, x_{3}, x_{4}, x_{5}, x_{6}\right)=\overline{x_{1}} \overline{x_{2}} x_{3} \overline{x_{5}} \overline{x_{6}}+\overline{x_{1}} \overline{x_{2}} x_{4} x_{5} \overline{x_{6}}+\overline{x_{1}} x_{2} \overline{x_{4}} x_{5} \overline{x_{6}}+\overline{x_{1}} \overline{x_{2}} \overline{x_{3}} \overline{x_{4}} x_{6}+\overline{x_{1}} \overline{x_{2}} \overline{x_{4}} x_{5} x_{6}+$ $\overrightarrow{x_{1}} x_{2} \overline{x_{3}} x_{4}+\overline{x_{1}} x_{2} x_{3} \overline{x_{5}} x_{6}+\overline{x_{1}} x_{3} x_{4} \overline{x_{5}} x_{6}+x_{1} \overline{x_{2}} \overline{x_{3}} \overline{x_{5}} \overline{x_{6}}+x_{1} \overline{x_{2}} \overline{x_{4}} x_{5} \overline{x_{5}}+x_{1} x_{2} x_{3} \overline{x_{5}} \overline{x_{6}}+x_{1} x_{2} x_{4} x_{5} \overrightarrow{x_{5}}+$ $x_{1} \overrightarrow{x_{3}} x_{4} \overline{x_{5}} x_{6}+x_{1} \overline{x_{2}} x_{3} \overline{x_{4}} \overline{x_{5}} x_{6}+x_{5} x_{2} \overline{x_{3}} \overline{x_{1}} x_{6}+x_{1} x_{2} \bar{x}_{4} x_{5} x_{6}+x_{2} x_{3} x_{4} x_{5} x_{6}$

$S_{3}^{3}\left(x_{1}, x_{2}, x_{3}, x_{4}, x_{5}, x_{6}\right)=\overline{x_{1}} \overline{x_{2}} \overline{x_{4}} \overline{x_{5}} \overline{x_{6}}+\overline{x_{1}} \overline{x_{3}} x_{4} x_{5} \overline{x_{5}}+\overline{x_{1}} x_{3} \overline{x_{5}} \overline{x_{6}}+\overline{x_{1}} x_{3} x_{4} \overline{x_{5}}+\overline{x_{1}} x_{2} \overline{x_{3}} \overline{x_{4}} \overline{x_{5}} x_{6}+$ $\overrightarrow{x_{1}} x_{2} x_{3} \bar{x}_{4} x_{5} x_{6}+x_{1} \overline{x_{2}} \overline{x_{4}} x_{5} \overline{x_{6}}+\overline{x_{2}} x_{3} \overline{x_{4}} x_{5} \overline{x_{6}}+x_{1} x_{2} \overline{x_{3}} \bar{x}_{5} \overline{x_{5}}+x_{3} x_{4} \overline{x_{5}} \overline{x_{6}}+x_{1} x_{2} x_{3} x_{5} \overline{x_{5}}+\bar{x}_{2} \overline{x_{3}} \overline{x_{4}} x_{5} x_{6}+$ $\overline{x_{2}} x_{3} \overline{x_{4}} \overline{x_{5}} x_{6}+\overline{x_{2}} x_{3} x_{4} x_{5} x_{6}+x_{1} \overline{x_{3}} \overline{x_{4}} x_{5} x_{6}+x_{1} x_{2} x_{4} \overline{x_{5}}+x_{2} \overline{x_{3}} x_{4} x_{5} x_{6}+x_{1} x_{3} \overline{x_{4}} \overline{x_{5}} x_{6}$

$S_{4}^{3}\left(x_{1}, x_{2}, x_{3}, x_{4}, x_{5}, x_{6}\right)=\overline{x_{2}} \overline{x_{2}} x_{4} \overline{x_{5}} \overline{x_{6}}+\overline{x_{1}} \overline{x_{2}} x_{3} x_{5} \overline{x_{5}}+\overline{x_{1}} x_{2} \overline{x_{3}} \bar{x}_{5} \overline{x_{6}}+\overline{x_{1}} x_{2} \bar{x}_{3} x_{5} x_{6}+\overline{x_{1}} \overline{x_{2}} \overline{x_{4}} \overline{x_{5}} x_{6}+$ $\overline{x_{1}} x_{2} x_{4} \overline{x_{5}} x_{6}+\overline{x_{1}} x_{2} x_{3} x_{5} x_{6}+\overline{x_{1}} \overline{x_{3}} \overline{x_{4}} \overline{x_{5}} \overline{x_{6}}+x_{1} \overline{x_{2}} \overline{x_{3}} x_{4} \bar{x}_{5} \overline{x_{6}}+\overline{x_{2}} x_{3} \overline{x_{4}} x_{5} \overline{x_{5}}+\overline{x_{2}} x_{3} x_{4} \overline{x_{3}} \overline{x_{6}}+$ $x_{2} \overline{x_{3}} \overline{x_{4}} \overline{x_{6}}+x_{2} \overline{x_{4}} \overline{x_{5}} \overline{x_{6}}+x_{1} x_{2} x_{3} x_{4} x_{5} \overline{x_{6}}+x_{2} \overline{x_{2}} \overline{x_{3}} \overline{x_{5}} x_{6}+x_{1} \overline{x_{2}} x_{3} x_{5} x_{5}+x_{1} x_{2} \overline{x_{3}} x_{5} x_{6}+x_{1} x_{2} x_{3} \overline{x_{4}} x_{6}$

$S_{1}^{4}\left(x_{1}, x_{2}, x_{3}, x_{4}, x_{5}, x_{6}\right)=\overline{x_{1}} \overline{x_{2}} \overline{x_{3}} \overline{x_{4}} x_{5}+\overline{x_{1}} \overline{x_{2}} x_{4} \overline{x_{5}} \overline{x_{6}}+\overline{x_{1}} \overline{x_{3}} x_{4} \overline{x_{5}} \overline{x_{6}}+\overline{x_{1}} x_{2} x_{3} \overline{x_{4}} \overline{x_{6}}+\overline{x_{1}} x_{3} x_{4} x_{5} \overline{x_{6}}+$ $\overline{x_{1}} \overline{x_{2}} \overline{x_{3}} \overline{x_{5}} x_{6}+\overline{x_{1}} x_{3} \overline{x_{4}} x_{5} x_{6}+\overline{x_{1}} x_{2} x_{3} x_{4} x_{6}+\overline{x_{2}} \overline{x_{3}} x_{4} \overline{x_{5}} \overline{x_{6}}+x_{1} \overline{x_{2}} \overline{x_{4}} \overline{x_{5}} \overline{x_{6}}+x_{1} \overline{x_{2}} x_{3} x_{5} \overline{x_{6}}+\bar{x}_{1} \overline{x_{3}} \overline{x_{4}} \overline{x_{5}} \overline{x_{6}}+$ $x_{1} x_{2} \overline{x_{3}} x_{4} x_{5}+x_{1} x_{2} x_{3} x_{4} \overline{x_{5}} \overline{x_{6}}+\overline{x_{2}} \overline{x_{3}} \overline{x_{4}} x_{5} x_{6}+x_{1} \overline{x_{2} x_{3}} \overline{x_{5}} x_{6}+x_{1} x_{3} x_{4} x_{5} x_{6}+x_{2} x_{2} \bar{x}_{4} \bar{x}_{5} x_{6}+$ $x_{2} x_{4} x_{5} x_{6}$

$S_{2}^{4}\left(x_{1}, x_{2}, x_{3}, x_{4}, x_{5}, x_{5}\right)=\overline{x_{1}} \overline{x_{2}} \overline{x_{3}} \overline{x_{5}} \overline{x_{6}}+\overline{x_{1}} x_{3} \overline{x_{4}} x_{5} \overline{x_{5}}+\overline{x_{1}} \overline{x_{2}} x_{3} \overline{x_{4}} x_{6}+\overline{x_{1}} \overline{x_{3}} \overline{x_{4}} \overline{x_{5}} x_{6}+\overline{x_{1}} \overline{x_{3}} x_{4} x_{5} x_{6}+$ $\overline{x_{1}} x_{2} x_{3} x_{4} \overline{x_{5}}+\overline{x_{2}} \overline{x_{3}} \overline{x_{4}} x_{5} \overline{x_{6}}+x_{1} x_{3} x_{4} x_{5} \overline{x_{6}}+x_{1} x_{2} \overline{x_{4}} \overline{x_{5}} \overline{x_{6}}+x_{1} x_{3} \overline{x_{4}} \overline{x_{5}} \overline{x_{6}}+x_{2} x_{4} x_{5} \overline{x_{6}}+x_{1} \overline{x_{3}} \overline{x_{4}} x_{5} x_{6}+$ $\overline{x_{2}} \overline{x_{3}} x_{4} x_{5} x_{6}+x_{1} \bar{x}_{2} x_{3} x_{4} \overline{x_{5}}+x_{2} \overline{x_{3}} \overline{x_{4}} x_{5} x_{6}+x_{1} x_{2} x_{3} x_{4} \overline{x_{5}} x_{6}+x_{1} x_{2} x_{3} \overline{x_{4}} x_{6}+x_{1} x_{2} x_{3} x_{5} x_{6}$

$S_{3}^{4}\left(x_{1}, x_{2}, x_{3}, x_{4}, z_{5}, x_{5}\right)=\overline{x_{1}} \overline{x_{2}} \overline{x_{3}} \overline{x_{5}} \overline{x_{6}}+\overline{x_{1}} \overline{x_{2}} x_{4} x_{5} \overline{x_{6}}+\overline{x_{1}} x_{2} \overline{x_{3}} \overline{x_{4}} x_{5}+\overline{x_{1}} x_{2} x_{3} \overline{x_{4}} \overline{x_{5}} \overline{x_{5}}+$ $\bar{x}_{1} x_{3} x_{4} x_{5} \overline{x_{6}}+\overline{x_{2}} \overline{x_{2}} x_{3} x_{5}+\overline{x_{1}} \overline{x_{3}} x_{4} \overline{x_{5}} x_{6}+x_{1} \overline{x_{2}} \overline{x_{4}} x_{5} \overline{x_{6}}+x_{1} \overline{x_{2}} x_{3} x_{4} \overline{x_{5}} \overline{x_{6}}+x_{2} \overline{x_{3}} \overline{x_{4}} \overline{x_{5}} \overline{x_{6}}+x_{1} x_{2} \bar{x}_{3} x_{4} \overline{x_{6}}+$ $x_{1} x_{3} \overline{x_{4}} x_{5} \overline{x_{6}}+x_{1} \overline{x_{2}} \overline{z_{4}} \overline{x_{5}} x_{6}+x_{1} \overline{x_{2}} \overline{x_{3}} x_{5} x_{6}+\overline{x_{2}} x_{3} \overline{x_{4}} \overrightarrow{x_{5}} x_{6}+x_{2} x_{3} \overline{x_{4}} x_{5} x_{6}+x_{2} x_{3} \bar{x}_{4} \overline{x_{5}} x_{6}+x_{1} x_{2} x_{4} x_{5} x_{6}$

$S_{4}^{4}\left(x_{1}, x_{2}, x_{3}, x_{4}, x_{5}, x_{6}\right)=\overline{x_{1}} \overline{x_{3}} \overline{x_{4}} \overline{x_{5}} \overline{x_{6}}+\overline{x_{1}} \overline{x_{2}} \overline{x_{3}} x_{5} \overline{x_{5}}+\overline{x_{1}} x_{2} x_{4} \bar{x}_{5} \overline{x_{6}}+\overline{x_{1}} \overline{x_{2}} \overline{x_{3}} \overline{x_{5}} x_{6}+\overline{x_{1}} \overline{x_{2}} x_{4} x_{5} x_{6}+$

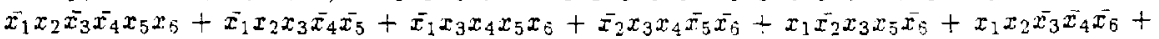
$x_{1} \overline{x_{3}} x_{4} \overline{x_{5}} \overline{x_{6}}+x_{2} \overline{x_{4}} \overline{x_{5}} \overline{x_{6}}+x_{1} \overline{x_{2}} \overline{x_{4}} x_{5} x_{6}+\overline{x_{2}} x_{3} \overline{x_{4}} x_{5} x_{6}+x_{1} \overline{x_{2}} x_{3} x_{4} \overline{x_{5}}+x_{1} \overline{x_{3}} \overline{x_{4}} \overline{x_{5}} x_{5}+x_{2} x_{2} \overline{x_{3}} x_{4} x_{6}+$ $x_{1} x_{3} \overline{x_{4}} x_{5} x_{6}$

$S_{1}^{5}\left(x_{1}, x_{2}, x_{3}, x_{4}, x_{5}, x_{6}\right)=\overline{x_{1}} \overline{x_{2}} \overline{x_{4}} x_{5} \overline{x_{5}}+\overline{x_{1}} x_{2} x_{1} x_{5} \overline{x_{6}}+\overline{x_{1}} x_{2} \overline{x_{4}} \overline{x_{5}} \overline{x_{6}}+\overline{x_{1}} \overline{x_{3}} x_{4} x_{5} x_{6}+\overline{x_{1}} x_{2} x_{4} \overline{x_{5}} x_{6}+$ $\overline{x_{1}} x_{2} x_{3} \overline{x_{4}} x_{5} x_{6}+\overline{x_{1}} x_{3} x_{4} \overline{x_{5}}+x_{1} \overline{x_{2}} x_{4} x_{5} \overline{x_{6}}+x_{1} \overline{x_{2}} x_{3} \overline{x_{4}} \overline{x_{6}}+x_{1} \bar{x}_{2} \overline{x_{3}} \overline{x_{4}} \overline{x_{6}}+x_{1} x_{2} \overline{x_{3}} \overline{x_{5}} \overline{x_{6}}+x_{2} x_{3} x_{4} x_{5} \overline{x_{6}}+$ $\overline{x_{2}} \overline{x_{3}} \overrightarrow{x_{4}} x_{6}+x_{1} \overline{x_{2}} \overline{x_{3}} \overline{x_{5}} x_{5}+x_{1} \overline{x_{2}} x_{3} x_{5}+\bar{x}_{1} \overline{x_{3}} \overline{x_{4}} x_{5} x_{6}+\mathrm{r}_{2} \overline{x_{3} x_{4} x_{5}} \mathrm{x}_{6}+x_{1} x_{2} x_{3} \overline{x_{4}} \overline{x_{5}} x_{6}$

$S_{2}^{5}\left(x_{1}, x_{2}, x_{3}, x_{4}, x_{5}, x_{6}\right)=\overline{x_{1}} \overline{x_{2}} x_{3} x_{4} \overline{x_{5}} \overline{x_{6}}+\overline{x_{2}} x_{3} \overline{x_{4}} \overline{x_{5}} \overline{x_{6}}+\overline{x_{1}} \overline{x_{2}} x_{3} x_{4} x_{5} \overline{x_{5}}+\overline{x_{1}} \overline{x_{3}} \overline{x_{4}} x_{5} \overline{x_{6}}+$ $\overline{x_{1}} x_{2} x_{3} \overrightarrow{x_{5}} \overline{x_{6}}+\overrightarrow{x_{1}} \overline{x_{2}} \overline{x_{4}} \overline{x_{5}} x_{6}+\overline{x_{1}} \overline{x_{2}} x_{3} \overline{x_{5}} x_{6}+\overrightarrow{x_{1}} x_{2} \overline{x_{3}} \overline{x_{5}} x_{6}+\overrightarrow{x_{1}} x_{2} x_{3} x_{4} x_{5} x_{6}+\overline{x_{1}} \overline{x_{2}} x_{3} \overline{x_{4}} x_{5}+$ $x_{1} \overline{x_{2}} x_{3} x_{4} \overline{x_{5}} \overline{x_{6}}+x_{1} \overline{x_{3}} \overline{x_{4}} \overline{x_{5}} \overline{x_{6}}+x_{1} x_{2} \overline{x_{3}} \overline{x_{5}} \overline{x_{6}}+x_{2} \bar{x}_{3} x_{4} x_{5} \overline{x_{6}}+x_{2} x_{3} \overline{x_{4}} \overline{x_{5}} \overline{x_{6}}+x_{1} x_{2} x_{4} x_{5} \overline{x_{6}}+$ $x_{1} \overline{x_{2}} \overline{x_{3}} x_{4} x_{6}+\overline{x_{2}} \overline{x_{3}} x_{4} x_{5} x_{6}+\bar{x}_{2} x_{3} \overline{x_{4}} x_{5} x_{6}+x_{1} \overline{x_{2}} x_{4} x_{5} x_{6}+x_{2} \overrightarrow{x_{3}} \overline{x_{4}} \overline{x_{5}} x_{6}+x_{1} x_{2} \bar{x}_{4} x_{5} x_{5}+x_{1} x_{2} x_{3} x_{4} \overline{x_{5}} x_{6}$

$S_{3}^{5}\left(x_{1}, x_{2}, x_{3}, x_{4}, x_{5}, x_{6}\right)=\overline{x_{1}} \overline{x_{2}} \overline{x_{4}} \overline{x_{5}} \overline{x_{6}}+\overline{x_{1}} \overline{x_{2}} x_{3} \overline{x_{6}}+\overline{x_{1}} x_{2} \bar{x}_{3} x_{4}+\overline{x_{1}} x_{3} x_{4} \overline{x_{5}} x_{6}+\overline{x_{2}} \overline{x_{2}} \overline{x_{4}} x_{5} x_{6}+$ 
$\overline{x_{1}} \overline{x_{3}} x_{4} \overline{x_{5}} x_{6}+x_{1} \overline{x_{2}} \overline{x_{3}} x_{5} \overline{x_{6}}+x_{1} x_{2} \overline{x_{4}} \overline{x_{5}}+x_{1} x_{3} \overline{x_{4}} \overline{x_{5}} \overline{x_{6}}+x_{1} x_{2} x_{3} x_{5} \overline{x_{6}}+\overline{x_{2}} \overline{x_{3}} \overline{x_{4}} \overline{x_{5}} x_{6}+x_{1} \overline{x_{2}} \overline{x_{3}} x_{4} x_{5}+$ $\overline{x_{2}} x_{3} \overline{x_{4}} x_{5} x_{6}+x_{1} \overline{x_{2}} x_{3} x_{4} \overline{x_{5}}+x_{1} x_{2} \overline{x_{3}} \overline{x_{4}} x_{6}+x_{2} x_{3} \overline{x_{4}} \overline{x_{5}} x_{6}+x_{2} x_{3} x_{4} x_{5} x_{6}$

$S_{4}^{5}\left(x_{1}, x_{2}, x_{3}, x_{4}, x_{5}, x_{6}\right)=\overline{x_{1}} x_{2} \overline{x_{3}} x_{4} \overline{x_{6}}+\overrightarrow{x_{1}} x_{3} \overrightarrow{x_{4}} \overline{x_{5}} \overrightarrow{x_{5}}+\overline{x_{1}} x_{2} x_{4} x_{5} \overline{x_{6}}+\overline{x_{1}} \overline{x_{2}} \overline{x_{4}} x_{5} x_{6}+\overrightarrow{x_{1}} \overline{x_{2}} x_{3} x_{4} x_{6}+$ $\overline{x_{1}} x_{2} \overline{x_{3}} \overline{x_{5}} x_{6}+\overline{x_{1}} x_{2} \overline{x_{4}} \overline{x_{5}} x_{6}+\overline{x_{1}} x_{3} \overline{x_{4}} x_{5} x_{6}+x_{1} \overline{x_{2}} x_{4} \overline{x_{5}} \overline{x_{6}}+\overline{x_{2}} x_{3} x_{4} \overline{x_{5}} \overline{x_{6}}+x_{1} x_{2} \overline{x_{3}} \overline{x_{4}} \overline{x_{6}}+x_{2} \overline{x_{3}} x_{5} \overline{x_{6}}+$ $\overline{x_{3}} x_{4} x_{5} \overline{x_{6}}+x_{1} x_{3} \overline{x_{4}} x_{5} \overline{x_{6}}+x_{1} \overline{x_{2}} \overline{x_{4}} \overline{x_{5}} x_{6}+x_{1} x_{2} \overline{x_{3} x_{5}}+x_{1} x_{2} x_{3} x_{4} x_{6}+x_{1} x_{4} x_{5} x_{6}$

$S_{1}^{6}\left(x_{1}, x_{2}, x_{3}, x_{4}, x_{5}, x_{6}\right)=\overline{x_{1}} \overline{x_{2}} x_{4} x_{5} \overline{x_{6}}+\overline{x_{1}} x_{2} \overline{x_{3}} \overline{x_{4}} x_{5} \overline{x_{6}}+\overline{x_{1}} x_{3} \overrightarrow{x_{4}} \overline{x_{5}} \overline{x_{6}}+\overline{x_{1}} x_{3} x_{4} x_{5} \overline{x_{6}}+$ $\overline{x_{1}} \overline{x_{2}} \overline{x_{3}} \overline{x_{4}} x_{6}+\overline{x_{1}} x_{2} \overline{x_{3}} x_{4} x_{6}+\overline{x_{1}} x_{3} \overline{x_{4}} x_{5} x_{6}+x_{1} \overline{x_{2}} \overline{x_{4}} x_{5} \overline{x_{6}}+\overline{x_{2}} \overline{x_{3}} \overline{x_{5}} \overline{x_{6}}+x_{1} x_{2} \overline{x_{3}} x_{4} x_{5} \overline{x_{6}}+x_{1} x_{3} \overline{x_{4}} x_{5} \overline{x_{6}}+$ $x_{1} x_{3} x_{4} \overline{x_{5}}+x_{1} \overline{x_{2}} x_{4} x_{5} x_{6}+x_{1} \overline{x_{2}} x_{3} \overline{x_{5}} x_{6}+\overline{x_{2}} x_{3} x_{4} \overline{x_{5}} x_{6}+x_{1} x_{2} \overline{x_{3}} \overline{x_{4}} x_{6}+x_{2} x_{3} x_{4} x_{5} x_{6}$

$S_{2}^{6}\left(x_{1}, x_{2}, x_{3}, x_{4}, x_{5}, x_{6}\right)=\overline{x_{1}} \overline{x_{2}} \overline{x_{3}} \overline{x_{4}} \overline{x_{5}} \overline{x_{6}}+\overline{x_{1}} x_{2} \overline{x_{4}} x_{5} \overline{x_{6}}+\overline{x_{1}} \overline{x_{3}} x_{4} x_{5} \overline{x_{6}}+\overline{x_{1}} x_{2} x_{3} \overline{x_{5}} \overline{x_{6}}+$ $\overline{x_{1}} \overline{x_{2}} \overline{x_{4}} x_{5} x_{6}+\overline{x_{1}} \overline{x_{2}} x_{3} \overline{x_{4}} x_{6}+\overline{x_{1}} \overline{x_{2}} x_{3} x_{5} x_{6}+\overline{x_{1}} x_{2} \overline{x_{3}} \overline{x_{5}} x_{6}+\overrightarrow{x_{1}} \overline{x_{3}} x_{4} \overline{x_{5}} x_{6}+x_{1} \overline{x_{2}} \overline{x_{3}} x_{5} \overline{x_{6}}+\overline{x_{2}} x_{3} x_{4} \overline{x_{5}} \overline{x_{6}}+$ $x_{1} x_{2} \overline{x_{3}} \overline{x_{5}} \overline{x_{6}}+x_{1} \overline{x_{3}} x_{4} \overline{x_{5}} \overline{x_{6}}+x_{1} x_{2} x_{3} x_{5} \overline{x_{6}}+x_{1} \overline{x_{2}} \overline{x_{3}} \overline{x_{4}} \overline{x_{5}} x_{6}+x_{1} \overline{x_{3}} x_{4} x_{5} x_{6}+\overline{x_{2}} x_{3} \overline{x_{4}} x_{5} x_{6}+$ $x_{1} \overline{x_{2}} x_{3} x_{4} \overline{x_{5}}+x_{1} x_{2} \overline{x_{3}} x_{5} x_{6}+x_{2} \overline{x_{3}} x_{4} x_{5} x_{6}+x_{1} x_{2} x_{3} \bar{x}_{4} \overline{x_{5}} x_{6}+x_{1} x_{2} x_{4} x_{5} x_{5}$

$S_{3}^{6}\left(x_{1}, x_{2}, x_{3}, x_{4}, x_{5}, x_{6}\right)=\overline{x_{1}} \bar{x}_{2} \overline{x_{3}} x_{4} \bar{x}_{6}+x_{1} x_{2} x_{4} \overline{x_{5}} \overline{x_{6}}+\overline{x_{1}} \overline{x_{3}} x_{4} \overline{x_{5}} x_{6}+x_{1} x_{2} x_{3} \overline{x_{4}} \overline{x_{6}}+\overline{x_{1}} x_{3} \bar{x}_{4} x_{5} x_{5}+$

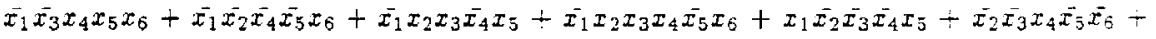
$x_{1} \overline{x_{2}} x_{3} \overline{x_{4}} \overline{x_{5}} \overline{x_{6}}+x_{1} x_{3} x_{4} x_{5} \overline{x_{6}}+x_{1} x_{2} \overline{x_{3}} \overline{x_{4}} \overline{x_{5}}+x_{1} x_{2} x_{4} x_{5} \overline{x_{6}}+x_{1} x_{2} x_{3} x_{4} \overline{x_{6}}+x_{2} x_{3} x_{4} x_{5} \overline{x_{5}}+$ $\overline{x_{2}} \overline{x_{3}} \overline{x_{4}} x_{5} x_{6}+x_{1} \overline{x_{2}} x_{4} \overline{x_{5}} x_{6}+x_{1} \overline{x_{2}} x_{3} x_{4} x_{5}+x_{2} \overline{x_{3}} \overline{x_{4}} \overline{x_{5}} x_{6}+x_{1} \overline{x_{3}} \overline{x_{4}} x_{5} x_{6}+x_{2} \overline{x_{3}} x_{4} x_{5} \bar{x}_{6}+x_{1} x_{2} \overline{x_{4}} \overline{x_{5}} x_{5}$

$S_{4}^{6}\left(x_{1}, x_{2}, x_{3}, x_{4}, x_{5}, x_{6}\right)=\overrightarrow{x_{1}} \overrightarrow{x_{2}} x_{3} \overrightarrow{x_{4}} \overrightarrow{x_{5}}+\overrightarrow{x_{1}} x_{2} x_{4} \overrightarrow{x_{5}}+\overrightarrow{x_{1}} x_{2} x_{3} x_{5} \bar{x}_{6}+\overrightarrow{x_{1}} \overline{x_{2}} x_{3} x_{4} x_{6}+\overrightarrow{x_{1}} \overline{x_{3}} \overline{x_{4}} x_{5}+$ $\overline{x_{1}} x_{2} \overrightarrow{x_{4}} x_{5}+x_{1} \overline{x_{2}} \overrightarrow{x_{3}} \overrightarrow{x_{5}} \overline{x_{6}}+\overline{x_{2}} \overline{x_{3}} x_{4} x_{5} \overline{x_{6}}+x_{1} \overrightarrow{x_{2}} x_{4} x_{5} \overline{x_{6}}+x_{1} x_{2} \overrightarrow{x_{4}} \overrightarrow{x_{5}} \overline{x_{6}}+x_{2} x_{3} \overline{x_{4}} x_{5} \overline{x_{6}}+x_{2} x_{3} x_{4} \overline{x_{5}} \overline{x_{6}}+$ $x_{1} \overline{x_{2}} \overline{x_{4}} x_{5} x_{6}+\overline{x_{2}} x_{3} \overline{x_{5}} x_{6}+x_{1} x_{2} \overline{x_{3}} \overline{x_{5}} x_{6}+x_{1} x_{2} x_{4} x_{5} x_{6}$

$S_{1}^{7}\left(x_{1}, x_{2}, x_{3}, x_{4}, x_{5}, r_{5}\right)=\overline{x_{1}} \overline{x_{3}} \overline{x_{4}} x_{5} \overline{x_{6}}+\overline{x_{1}} \overline{x_{2}} x_{3} \overline{x_{5}} \overline{x_{5}}+\overline{x_{1}} x_{2} \overline{x_{3}} x_{4} \bar{x}_{5} \overline{x_{6}}+x_{1} x_{2} \overline{x_{4}} x_{5} \overline{x_{6}}+$ $\overline{x_{1}} \overline{x_{3}} \overline{x_{4}} \overline{x_{5}} x_{6}+\overline{x_{1}} \overline{x_{2}} x_{3} x_{5} x_{6}+\overline{x_{1}} x_{3} \overline{x_{4}} x_{5} x_{5}+\bar{x}_{1} x_{2} x_{3} x_{4} \overline{x_{5}} x_{6}+x_{1} \overline{x_{2}} \overline{x_{3}} x_{4}+\overline{x_{2}} x_{3} \overline{x_{4}} \overline{x_{5}} \overline{x_{5}}+x_{2} x_{4} x_{5} \overline{x_{5}}+$ $x_{1} x_{2} \overline{x_{3}} \overline{x_{4}} \overline{x_{6}}+x_{1} x_{2} x_{3} x_{4} \overline{x_{5}} \overline{x_{6}}+x_{1} \overline{x_{2}} \overline{x_{3}} x_{5} x_{5}+\overline{x_{2}} \overline{x_{3}} x_{4} \overline{x_{5}} x_{6}+x_{1} \overline{x_{3}} x_{4} x_{5}+x_{1} \overline{x_{2}} x_{4} \overline{x_{5}} x_{6}+x_{2} \overline{x_{3}} x_{4} x_{5} x_{5}-$ $x_{1} x_{2} \overline{x_{4}} \overline{x_{5}} x_{6}+x_{1} x_{2} x_{4} x_{5} x_{6}$

$S_{2}^{7}\left(x_{1}, x_{2}, x_{3}, x_{4}, x_{5}, x_{6}\right)=\overline{x_{1}} x_{2} x_{3} \overline{x_{5}} \overline{x_{5}}+\overline{x_{1}} \overline{x_{3}} x_{4} x_{5}+\overline{x_{1}} \overline{x_{2}} \overline{x_{4}} \overline{x_{5}}+\overline{x_{1}} x_{2} \overline{x_{3}} \overline{x_{5}} \overline{x_{6}}+\overline{x_{1}} x_{2} x_{3} x_{5} x_{5}+$ $x_{1} \overline{x_{3}} \overline{x_{4}} x_{5} \overline{x_{6}}+x_{1} \overline{x_{2}} x_{3} \overline{x_{5}} \overline{x_{6}}+\overline{x_{2}} x_{4} x_{5} \overline{x_{6}}+x_{2} x_{3} x_{4} x_{5} x_{6}+x_{1} x_{2} \bar{x}_{3} x_{4} \bar{x}_{5} x_{6}+x_{1} x_{2} \overline{x_{4}} x_{5} \bar{x}_{6}+x_{2} \overline{x_{2}} \overline{x_{3}} \overline{x_{5}} \bar{x}_{5}-$ $x_{1} \bar{x}_{2} x_{3} x_{5} x_{5}+x_{1} x_{2} \bar{x}_{3} x_{5} x_{6}+x_{1} x_{2} x_{3} \bar{x}_{4} \bar{x}_{5} x_{5}+x_{2} x_{4} x_{5} x_{6}$

$S_{3}^{7}\left(x_{1}, x_{2}, x_{3}, x_{4}, x_{5}, x_{5}\right)=\overline{x_{1}} \overline{x_{2}} \overline{x_{3}} x_{5} \overline{x_{5}}+\overline{x_{1}} \overline{x_{2}} x_{3} \overline{x_{4}} \overline{x_{5}} \overline{x_{6}}+\overline{x_{5}} \overline{x_{3}} x_{4} x_{5} \overline{x_{5}}+\overline{x_{1}} x_{2} \bar{x}_{3} \overline{x_{4}} x_{5}-$ $\overrightarrow{x_{1}} x_{2} x_{3} x_{4} \overline{x_{5}} \overrightarrow{x_{6}}+\overrightarrow{x_{1}} \overrightarrow{x_{2}} \overrightarrow{x_{3}} x_{4}+\overrightarrow{x_{1}} x_{2} \overline{x_{4}} x_{6}+\overrightarrow{x_{1}} x_{3} x_{4} x_{5} x_{5}+x_{1} \bar{x}_{2} x_{3} x_{5} \overline{x_{6}}+x_{1} \bar{x}_{2} x_{4} \overrightarrow{x_{5}} \overline{x_{6}}+x_{2} \overrightarrow{x_{3}} \overrightarrow{x_{4}} \overline{x_{5}} \overline{x_{0}}+$ $x_{1} x_{2} \overline{x_{3}} \overline{x_{4}} \overline{x_{6}}+x_{1} \overline{x_{3} x_{4}} \overline{x_{5}} \overline{x_{6}}+x_{1} x_{3} x_{4} x_{5} x_{6}+x_{1} \overline{x_{2}} x_{3} \overline{x_{4}} x_{6}+\overline{x_{2}} x_{3} x_{4} x_{5} x_{5}+x_{1} x_{2} \overline{x_{3}} x_{4} x_{5} x_{5}+$ $x_{2} x_{3} \bar{x}_{4} x_{6}+x_{1} x_{3} x_{4} x_{5} x_{6}$

$S_{4}^{7}\left(x_{1}, x_{2}, x_{3}, x_{4}, x_{5}, x_{6}\right)=\overline{x_{1}} \overline{x_{2}} \overline{x_{3}} \overline{x_{4}} x_{5} \overline{x_{5}}+\overrightarrow{x_{2}} x_{2} \overline{x_{3}} \overline{x_{5}} \overrightarrow{x_{6}}+\overrightarrow{x_{1}} x_{2} x_{4} x_{5} \overline{x_{6}}+\overline{x_{1}} x_{3} \overline{x_{4}} \overline{x_{5}} \bar{x}_{5}+$ $\overline{x_{1}} x_{3} x_{4} x_{5} \overline{x_{6}}+\overline{x_{1}} \overline{x_{2}} \overline{x_{3}} \overline{x_{5}} x_{5}+\overline{x_{1}} \overline{x_{2}} \overline{x_{3}} x_{4} x_{5}+\bar{x}_{1} \bar{x}_{2} x_{4} \overline{x_{5}} x_{6}+\overline{x_{1}} \overline{x_{3}} x_{4} \overline{x_{5}} x_{6}+\bar{x}_{1} x_{3} \overline{x_{4}} x_{5} x_{6}+x_{1} \overline{x_{2}} \overline{x_{3}} \overline{x_{5}} \overline{x_{5}}-$ $x_{1} \overline{x_{2}} \overline{x_{3}} x_{4} \overline{x_{6}}+x_{1} x_{2} \overline{x_{4}} x_{5} \overline{x_{6}}+x_{2} x_{3} \bar{x}_{4} x_{5} \bar{x}_{6}+x_{1} x_{3} x_{4} \overline{x_{5}} \overline{x_{6}}+x_{1} \overline{x_{3}} \overline{x_{4}} x_{5} x_{6}+\bar{x}_{2} \overline{x_{3}} \bar{x}_{4} \overline{x_{5}} x_{6}+x_{1} \overline{x_{2}} x_{3} \overline{x_{4}} \overline{x_{3}} x_{6}+$ $x_{1} \overline{x_{2}} x_{3} x_{4} x_{5} x_{6}+x_{1} x_{2} \overline{x_{3}} \overline{x_{4}} x_{6}+x_{2} \overline{x_{3}} \overline{x_{4}} x_{5} x_{6}+x_{1} x_{2} \overline{x_{3}} x_{5} x_{6}+x_{1} x_{2} x_{3} x_{4} \overline{x_{5}}$

$S_{1}^{8}\left(x_{1}, x_{2}, x_{3}, x_{4}, x_{5}, x_{6}\right)=\overline{x_{1}} \overline{x_{2}} x_{4} \overline{x_{5}} \overline{x_{6}}+\overline{x_{1}} \overline{x_{3}} \overline{x_{4}} \overline{x_{5}} \overline{x_{6}}+\overline{x_{1}} x_{2} \overline{x_{3}} \bar{x}_{5} \overline{x_{5}}+\overline{x_{1}} \overline{x_{2}} \overline{x_{3}} x_{5} x_{6}+\overline{x_{1}} \overline{x_{2}} x_{3} \overline{x_{4}} \overline{x_{5}} x_{5}+$ $\overline{x_{1}} \overline{x_{3}} x_{4} x_{5} x_{6}+\overline{x_{1}} x_{2} x_{3} \overline{x_{4}} x_{5} x_{6}+\overline{x_{1}} x_{2} x_{3} x_{4} x_{5}+x_{1} \bar{x}_{2} x_{4} x_{5} \overline{x_{6}}+\bar{x}_{2} x_{3} \bar{x}_{4} x_{5} \bar{x}_{6}+\overline{x_{2}} x_{3} x_{4} \overline{x_{3}} \overline{x_{0}}-$ $x_{1} x_{2} \bar{x}_{3} x_{4} \overline{x_{5}}+x_{1} x_{3} \overline{x_{4}} \overline{x_{5}} \overline{x_{6}}+x_{1} x_{2} x_{4} x_{5} \overline{x_{6}}+\overline{x_{2}} \overline{x_{3}} x_{4} \overline{x_{5}} x_{6}+x_{1} \overline{x_{2}} x_{3} x_{5} x_{6}-x_{2} \overline{x_{2}} x_{4} \overline{x_{5}} x_{5}+x_{2} \bar{x}_{3} \bar{x}_{4} \overline{x_{5}} x_{6}+$ $x_{1} x_{2} \bar{x}_{3} \bar{x}_{4} x_{6}+x_{1} \bar{x}_{3} x_{4} \bar{x}_{5} x_{5}+x_{1} x_{3} x_{4} x_{5} x_{5}$

$S_{2}^{8}\left(x_{1}, x_{2}, x_{3}, x_{4}, x_{5}, x_{6}\right)=\overline{x_{1}} \overline{x_{3}} x_{4} x_{5} \overline{x_{6}}+\overline{x_{1}} x_{3} \overline{x_{4}} \overline{x_{5}} \overline{x_{6}}+\overline{x_{1}} x_{2} x_{4} x_{5} \overline{x_{6}}+\overline{x_{1}} \overline{x_{3}} \overline{x_{4}} x_{5} x_{5}+\overline{x_{1}} \overline{x_{2}} x_{4} \overline{x_{5}} x_{6}+$

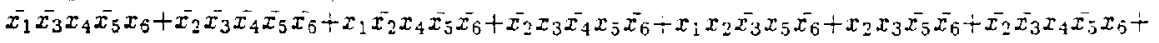
$x_{1} \bar{x}_{2} x_{4} x_{5} x_{6}+x_{1} \overline{x_{2}} x_{3} \overline{x_{4}} \overline{x_{3}} x_{6}+\overline{x_{2}} x_{3} x_{4} x_{5} x_{6}+x_{2} \overline{x_{3}} \overline{x_{4}} x_{6}+x_{2} \overrightarrow{x_{4}} x_{5} x_{5}+x_{1} x_{2} x_{3} x_{4} \overline{x_{5}}$

$S_{3}^{8}\left(x_{1}, x_{2}, x_{3}, x_{4}, x_{5}, x_{6}\right)=\overline{x_{1}} x_{2} \overline{x_{3}} \overline{x_{5}} \overline{x_{6}}+\overline{x_{1}} \overline{x_{2}} \overline{x_{4}} x_{5}+\overline{x_{1}} \overline{x_{2}} \bar{x}_{3} \overline{x_{5}}+\overline{x_{1}} x_{2} \overline{x_{3}} x_{4}+\overline{x_{1}} x_{2} x_{4} x_{5}+$

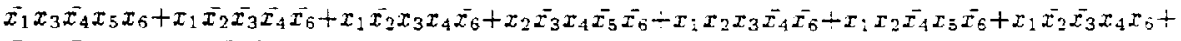
$\overline{x_{2}} x_{3} \overline{x_{4}} x_{5} x_{6}+x_{1} \overline{x_{3}} \overrightarrow{x_{4}} \overline{x_{5}} x_{6}+x_{1} x_{2} x_{3} \overline{x_{5}} x_{6}+x_{2} x_{3} x_{4} x_{5} x_{6}$

$S_{4}^{8}\left(x_{1}, x_{2}, x_{3}, x_{4}, x_{5}, x_{6}\right)=\overrightarrow{x_{1}} \overline{x_{2}} x_{3} x_{5} \overline{x_{5}}+\overline{x_{1}} \overline{x_{2}} x_{3} x_{4} \overline{x_{6}}+\overline{x_{1}} x_{2} \overline{x_{3}} \overline{x_{4}} x_{5}+\overline{x_{1}} \overline{x_{2}} \overline{x_{3}} x_{4} \overline{x_{5}} \overline{x_{6}}+$ $\overline{x_{1}} x_{3} x_{4} x_{5} \overline{x_{6}}+\overline{x_{1}} \overline{x_{2}} \overline{x_{3}} \overline{x_{3}} x_{6}+\overline{x_{1}} \overline{x_{2}} \overline{x_{4}} x_{5} x_{5}+\overline{x_{1}} x_{2} \overline{x_{3}} x_{5} x_{6}+\overline{x_{1}} x_{3} x_{4} \overline{x_{5}} x_{6}+\overline{x_{2}} \overline{x_{3}} \overline{x_{4}} \overline{x_{5}} \overline{x_{5}}+x_{1} \overline{x_{2}} \overline{x_{3}} x_{5}+$ $x_{1} x_{3} \overline{x_{4}} \overline{x_{5}} \overline{x_{6}}+x_{1} \overrightarrow{x_{3}} x_{4} x_{5} \overline{x_{6}}+x_{2} x_{3} \overline{x_{4}} \overline{x_{5}} \overrightarrow{x_{5}}+x_{1} x_{2} x_{3} \overline{x_{4}}+r_{1} x_{2} x_{3} \overline{x_{5}} \overline{x_{5}}+x_{1} x_{2} \overline{x_{3}} \overrightarrow{x_{5}} x_{6}+x_{1} x_{3} x_{4} x_{5} x_{6}$ 